Pacific Journal of Mathematics

FINITE TYPE EXTENSIONS AND COHERENCE 


\title{
FINITE TYPE EXTENSIONS AND COHERENCE
}

\author{
IRA J. PAPICK
}

\begin{abstract}
The purpose of this paper is to study the relationships between coherence, finite type extensions, and certain especial orderings on the spectrum of a commutative integral domain $R$. Applications of the techniques developed include a partial answer to a question of Vasconcelos concerning the integral closure of a 1-dimensional, local, coherent domain, the existence and construction of an interesting class of coherent domains that remain coherent under polynomial adjunction, and new characterizations of domains previously defined topologically.
\end{abstract}

1. A characterization of open Prüfer domains, Recall from [16] that $R$ is called a going-down domain (written $G D$ ) in case $R \subseteq T$ satisfies $G D$ for each domain $T$ satisfying $R \subseteq T \subseteq K$; an extension $R \cong T$ is called an $i$-extension if $\operatorname{Spec}(T) \rightarrow \operatorname{Spec}(R)$ is injective (unless otherwise specified, the map $\operatorname{Spec}(T) \rightarrow \operatorname{Spec}(R)$ is the contraction map), and say that $R$ is an $i$-domain if $R \subseteq T$ is an $i$-extension for each $R \cong T \subseteq K$; an extension $R \cong T$ is said to be open if $\operatorname{Spec}(T) \rightarrow \operatorname{Spec}(R)$ is open and $R$ is said to be open if $R \cong$ $T$ is open for each $R \cong T \subseteq K$. For $P, P^{\prime} \in \operatorname{Spec}(R)$ let $\left[P, P^{\prime}\right]=$ $\left\{Q \in \operatorname{Spec}(R): P \subseteq Q \subseteq P^{\prime}\right\}$. If $M$ is a maximal ideal of $R$, we call $[0, M]$ a branch of $R[16, \S 3]$. An especially useful characterization of open domains is as follows: [16, Theorem 3.16], $R$ is open if and only if $R$ is $G D$ and semilocal and each branch of $R$ is well-ordered under inclusion.

For an extension $R \subseteq T$, it is said that $T$ is of finite type over $R$ if there is an $R$-algebra homomorphism from a polynomial ring $R\left[x_{1}, \cdots, x_{n}\right]$ onto $T$, and furthermore, if the kernel of this homormophism is finitely generated, $T$ is said to be finitely presented as an $R$-algebra. Given a contraction map $f: \operatorname{Spec}(T) \rightarrow \operatorname{Spec}(R)$ and $P \in \operatorname{Spec}(R)$, denote the set $f^{-1}(P)$, the fiber of $P$, by fib $_{T}(P)[16, \S 4]$. A prime $Q \in \operatorname{Spec}(T)$ is said to be isolated in its fiber if $Q$ is maximal and minimal in $\mathrm{fib}_{T}(Q \cap R)$. A finite type extension $R \cong T$ is said to be quasi-finite at $Q \in \operatorname{Spec}(T)$, if $Q$ is isolated in its fiber, and $R \cong T$ is said to be quasi-finite if it is quasi-finite at each $Q €$ Spec $(T)$ [19, Definition 1, p. 40].

Any unexplained terminology is standard, as in [3] and [14].

Proposition 1. If each overring of $R$ is of finite type over $R$, then 
(a) each overring of $R$ is semilocal;

(b) each $G D$ overring of $R$ is open;

(c) $\bar{R}$ is an open Prïfer domain.

Proof. It suffices to show that $R$ is semilocal. For each $P \in$ Spec $(R), R \cong R_{P}$ is a flat extension of finite type, and thus $R_{P}$, is a finitely presented $R$-algebra [11, Corollaire (3.4.7)]. But [10, Proposition (7.3.10)] implies that $R \subseteq R_{P}$ is an open extension for each $P \in \operatorname{Spec}(R)$. An application of [16, Proposition 3.3] completes part (a).

(b) Let $T$ be a $G D$ overring of $R$. That $T$ is open is immediate from [16, Lemma 3.1]. (Notice that (b) also follows from [16, Theorem 3.16] with [16, Proposition 3.14].)

(c) This is a direct consequence of [16, Lemma 2.33] ([16, Proposition 2.26]).

We include an alternate proof of (c) which provides another view of the underlying ideas present. (This proof, combined with Theorem 2, shows that each valuation overring is of finite type iff each overring is of finite type, where $R$ is integrally closed.)

We may assume $R$ is integrally closed and local with maximal ideal $M$. Let $V$ be a dominating valuation overring of $R$ with maximal ideal $N$. Since $V$ is $G D$, (b) gives that $V$ is open, hence fib $_{V}(M)$ has a least element, say $P$. Thus, $R \cong V_{P}$ is quasi-finite at $P V_{P}$. Hence $R=R_{M}=\left(V_{P}\right)_{R \backslash M}[9$, Proposition 4, P. 43]. Whence, $R$ is a valuation domain, and the proof is complete [16, Corollary $3.17]$.

THEOREM 2. Let $R$ be integrally closed. The following are equivalent:

(a) $R$ is an open Prüfer domain.

(b) $R \cong T$ is simple for each $R \cong T \cong K$.

(c) $R \subseteq T$ is of finite type for each $R \subseteq T \subseteq K$.

(d) $R \subseteq T$ is finitely presented as an $R$-algebra for each $R \subseteq$ $T \cong K$.

Proof. $(\mathrm{b}) \Rightarrow$ (c) is clear. Proposition 1 (c) combined with [11, Corollaire (3.4.7)] shows that $(\mathrm{c}) \Rightarrow(\mathrm{d})$. Another application of Proposition $1(\mathrm{c})$ gives $(\mathrm{d}) \Rightarrow(\mathrm{a})$. We focus our attention on $(\mathrm{a}) \Rightarrow(\mathrm{b})$. Consider $R \varsubsetneqq T \subseteq K$, and let $N_{1}, \cdots, N_{r}$ be the maximal ideals of $T$ [16, Corollary 4.12].

Let $W=\left\{P \in \operatorname{Spec}(R): P \varsubsetneqq N_{\imath} \cap R\right.$ for each $\left.1 \leqq i \leqq r\right\}$. We claim $W \neq \varnothing$. Suppose $W=\varnothing$. Thus each maximal ideal in $R$ equals $N_{i} \cap R$ for some $i$. Hence $R \cong T$ satisfies $L O$, since $R \subseteq T$ is 
flat and thus going-down. Therefore $R=T$ [20, Proposition 2], contradicting our original assumption. Let $Q=\bigcap_{P \in W} P$ and observe that $Q \nsubseteq \bigcup_{i=1}^{r} N_{i} \cap R$. For if $Q \subseteq \bigcup_{i=1}^{r} N_{i} \cap R$, then $Q \subseteq N_{j} \cap R$, some $j$, and an application of [18, Proposition 6] establishes that some $P \in W$ is contained in $N_{j} \cap R$, which is a contradiction. So, choose an $a \in Q \backslash \bigcup_{i=1}^{r}\left(N_{i} \cap R\right)$ and consider $R[1 / a]$. Since $a \notin N_{i}$ for each $i$, $1 / a \in T$ and therefore $R[1 / a] \leqq T$. To complete the proof we will show

$$
\operatorname{Max}(R[1 / a])=\left\{\left(N_{1} \cap R\right) R[1 / a], \cdots,\left(N_{r} \cap R\right) R[1 / a]\right\} .
$$

This being the case, we would have $R[1 / a]=T$, since $R[1 / a] \subseteq T$ is a flat extension satisfying $L O$ [20, Proposition 2].

Clearly each $N_{i} \cap R$ survives in $R[1 / a]$, and for $i \neq j, N_{i} \cap R \nsubseteq$ $N_{j} \cap R$ since $N_{i} \varsubsetneqq N_{j}$. If $\left(N_{i} \cap R\right) R[1 / a] \varsubsetneqq N$, where $N$ is maximal in $R[1 / a]$, then $N_{i} \cap R \varsubsetneqq N \cap R$, since $R \cong R[1 / a]$ is a flat extension. Thus $N \cap R \nsubseteq N_{i} \cap R$ for each $1 \leqq i \leqq r$; for if $N \cap R \subseteq N_{j} \cap R$ for some $j \neq i$, then $N_{i} \cap R \subseteq N_{j} \cap R$, a contradiction. Hence $Q \subseteq N \cap R$, which means a $\in N \cap R$, contradicting the existence of $N$. Therefore, $\left(N_{i} \cap R\right) R[1 / a]$ is maximal in $R[1 / a]$ for each $i$. In a similar fashion, one shows that these are the only maximal ideals of $R[1 / a]$, completing the proof.

EXAMPLE 3. (i) Theorem 2 is of course the integrally closed converse of Proposition 1(c). It is natural to ask if (a) and (b) of Proposition 1 have similar converses. We have not yet been able to answer this question relative to condition (b), however, with respect to condition (a) it is easy to find a counter-example. For example, let $V$ be a valuation ring whose spectrum is not wellordered under inclusion. Then, each overring of $V$ is local, yet Theorem 2 shows that some extension of $R$ is not of finite type over $R$.

(ii) We would not expect conditions (a), (b), or (c) to imply that each overring of $R$ is of finite type over $R$ for a nonintegrally closed $R$; moreover, we can find a nonintegrally closed $R$ satisfying (a), (b), and (c), yet having a nonfinite type extension. For example, let $R$ be a local, 1-dim., Noetherian domain such that $\bar{R}$ is not a finitely generated $R$-module. A direct appeal to [16, Proposition 3.20] shows that $R$ satisfies (a), (b), and (c).

REMARK AND EXAMPLE 4. If $R$ is a domain each of whose overrings is of finite type over $R$, we know (Proposition 1(c)) that $\bar{R}$ is an open Prüfer domain. It would be interesting as well as useful to know that $R$ was open. (In this situation, $R$ open is equivalent to $R G D$ [16, Proposition 3.22].) However, in general $R$ 
need not even be treed. We now produce such an $R$. (See [0, Lemmas 8 and 9] and [17, Example 16] for more details.) Let $k$ be an algebraically closed field, $x$ and $y$ indeterminates over $k$, and $F=k(x, y)$. Define the valuation rings $W=k+x W$ and $V=k+$ $y V$ as in [17, Example 2.28]. Let $T=V \cap W$ and $R=k+J(T)$, where $J(T)$ is the Jacobson radical of $T$. It follows, [8, Example 4.3], that each overring of $R$ distinct from $R$ contains $T$ and $\bar{R}=$ $T$. Hence $T$ is finitely generated as a module over $R$. But, Theorem 2 gives that each overring of $T$ is of finite type over $T$; hence, each overring of $R$ is of finite type over $R$, being a composition of a finite extension and a finite type extension.

REMARK 5. The domains of Theorem 2 ( $L H$-domains, (local homeomorphism)) arise naturally in another setting [16, Lemma 4.13]. For a topologically related study of such domains and their generalizations, see [16].

2. Coherence and finite type extensions. Recall [4] that a commutative ring is called coherent if each finitely generated ideal is finitely presented.

We start this section with a useful lemma, which in essence is a direct consequence of a beautiful theorem of Gruson and Raynaud.

In $\S 1$, we observed that in the integrally closed case, the assumption that each overring be of finite type over $R$, guaranteed the presence of coherence. Example 11 of this section shows that in the nonintegrally closed case, coherence is not always present. Thus, as some of our applications and interests deal specifically with coherent domains, we initiate in this short section the study of coherent domains $R$, each of whose overrings are of finite type over $R$.

LEMMA 6. Let $R \cong T$ be domains, and let $T$ be a finitely presented $R$-module. Then, $R$ is coherent if and only if $T$ is coherent.

Proof. The "only if" part follows directly from [12, Corollary 1.2]. For the converse, we use the criterion in [4, Theorem 2.1(a)]; i.e., we show that the product of any family $\left\{A_{j}: j \in J\right\}$ of flat $R$ modules is flat. As each $A_{j} \otimes_{R} T$ is $T$-flat and $T$ is coherent, $\Pi\left(A_{j} \otimes_{R} T\right)$ is $T$-flat. However, since $T$ is finitely presented over $R$, the canonical homomorphism $\left(\Pi A_{j}\right) \bigotimes_{R} T \rightarrow \Pi\left(A_{j} \bigotimes_{R} T\right)$ is an isomorphism [3, Exercise 9(a), p. 43], so that $\left(\Pi A_{j}\right) \bigotimes_{R} T$ is also $T$-flat. That $\Pi A_{j}$ is $R$-flat, now follows from the remarkable theorem of Gruson and Raynaud [11, Théorème (1.2.4)], and hence the proof is complete. 
REMARK 7. Example 11 shows that the assumption of finite presentation is needed in Lemma 6 . Lemma 6 can be viewed as the coherent analogue of Eakin's theorem [10, Proposition (6.4.9)], since the same proof shows the conclusion is valid for $R \cong T$ commutative rings with identity.

REMARK 8. Lemma 6 could be used in the proof of [6, Theorem 3] instead of applying Ferrand's descent result.

Proposition 9. If $R$ is coherent and each overring of $R$ is of finite type over $R$, then

(a) each overring of $R$ is coherent;

(b) each overring of $R$ is finitely presented as an $R$-algebra;

(c) $R\left[x_{1}, \cdots, x_{n}\right]$ is coherent.

Proof. Let $T$ be an arbitrary overring of $R$.

(a). Let $R^{\prime}$ be the integral closure of $R$ in $T$. Since $R \subseteq R^{\prime}$ is a finite extension, $R^{\prime}$ is coherent [12, Corollary 1.5]. [16, Proposition 2.26] combined with Proposition $1(\mathrm{c})$ implies that $R^{\prime} \subseteq T$ is a quasi-finite extension. Directly applying [19, Proposition 4, p. 43] and the proof of [20, Theorem 2] shows that $R^{\prime} \subseteq T$ is flat, and [9, Proposition 2.2] completes part (a).

(b). Again consider $R \cong R^{\prime} \subseteq T$. As $R$ is coherent and $R \subseteq R^{\prime} \leqq$ $K$, where $R^{\prime}$ is a finitely generated $R$-module, it is straightforward to verify that $R^{\prime}$ is a finitely presented $R$-module. Hence $R^{\prime}$ is a finitely presented $R$-algebra [10, Corollary (6.3.7)]. Also, as in (a), $R^{\prime} \subseteq T$ is flat and of finite type, so $T$ is a finitely presented $R^{\prime}$-algebra [11, Corollaire (3.4.7)]. Therefore, $T$ is a finitely presented $R$-algebra $[10,(6.3 .5)]$.

(c). By Proposition 1 (c) we have $\bar{R}$ Prüfer, hence $\bar{R}\left[x_{1}, \cdots, x_{n}\right]$ is coherent [21, Corollary (4.6)]. However, $\bar{R}\left[x_{1}, \cdots, x_{n}\right]$ is a finitely presented $R\left[x_{1}, \cdots, x_{n}\right]$-module [10, (6.3.5), Proposition (I, 6. 2.10)], so Lemma 6 implies that $R\left[x_{1}, \cdots, x_{n}\right]$ is coherent, completing the proof.

EXAMPle AND a Converse 10. It would indeed be nice if conditions (a), (b), or (c) of Proposition 9 were sufficient as well as necessary. This is the case for (b), yet is not true for (a) and (c). The example in 3(ii) provides a domain satisfying (a) and (c) but having a nonfinite type extension. If we try to achieve converses 
of (a) and (c) by imposing an integrally closed condition, we still do not succeed. For let $R$ be a nonopen Prüfer domain, e.g., the integers. Then certainly conditions (a) and (c) are satisfied, yet Theorem 2 shows that $R$ must have a nonfinite type extension.

We now show that (b) is sufficient in Proposition 9. Clearly, it is enough to show that $R$ is coherent. Proposition 1(c) implies that $\bar{R}$ is coherent and we are assuming $\bar{R}$ is a finitely presented $R$-module. Hence, by Lemma 6 , we conclude that $R$ is coherent.

Finally, we mention that Proposition 9(c) combined with a standard direct limit argument [3, Exercise 12e, p. 44] shows that the ring of polynomials in an arbitrary number of indeterminates over a domain $R$ satisfying the hypothesis of Proposition 9 is coherent.

EXAMPLE 11. The following example shows that the coherence assumption is needed in Proposition 9, i.e., we construct a noncoherent domain $R$ each of whose overrings are of finite type over $R$. Let $L$ be a finite field extension of the field $k$, and let $V$ be an open valuation ring of the form $L+M$, where $M$ is the maximal ideal of $V$ and is nonfinitely generated. (See [6] and [17] for specific construction.) Let $R=k+M$. Then, [6, Theorem 3] shows that $R$ is not coherent, yet each overring of $R$ is of finite type over $R$, since each compares with $V$ [1, Theorem 3.1].

EXAmple 12. The purpose of this example is to present the reader with an interesting class of domains satisfying the hypothesis of Proposition 9. As in Example 11, let $[L: k]<\infty$ and let $V=L+M$, but in this case take $M$ to be finitely generated. Then, [6, Theorem 3] shows $R=k+M$ is coherent and as in Example 11, each overring of $R$ is of finite type over $R$.

3. Very finite extensions. In this final section we give a positive partial answer to a question of Vasconcelos, namely: is the integral closure of a 1-dimensional, local coherent domain a Prüfer domain? We also give some applications to $\S \S 1$ and 2.

We call an extension $R \varsubsetneqq T$ where $T$ is an overring of $R$ and $T$ is a finitely generated $R$-module, a very finite extension if $S$ is a finitely generated $R$-module for each overring $S$ satisfying $R \cong$ $S \subseteq T$. Examples of such extensions are module-finite overrings of arbitrary Noetherian domains, finite minimal homomorphisms as defined in [7] and extensions arising via the $D+M$-construction (e.g., see Example 11). Recall, that an extension $R \subseteq T$ is called an $L H$-extension if the contraction map $\operatorname{Spec}(T) \rightarrow \operatorname{Spec}(R)$ is a local homeomorphism, and $R$ is called an $L H$-domain if $R \subseteq T$ is an $L H$ - 
extension for each overring $T$ of $R[16, \S 4]$.

Lemma 13. Let $R \subseteq S \subseteq T$ be domains such that $T$ is a finitely presented $R$-module. Then, $T$ is a finitely presented $S$-module if and only if $S$ is a finitely generated $R$-module.

Proof. The "if" part follows from [13, II. Appendix F. 4] and [10, Proposition (6.2.10)]. As for the "only if" part, let $I$ be a set with $\operatorname{card}(I)=\operatorname{card}(S)$, and for each $i \in I$, let $R_{i}=R$. A straightforward calculation shows that for $S$ to be a finitely generated $R$ module it is sufficient to show that the canonical map $\left(\Pi R_{i}\right) \bigotimes_{R} S \rightarrow$ $\Pi\left(R_{\imath} \otimes_{R} S\right)$ is onto. (It is also necessary, [2, Exercise 3(a), p. 396].) Let $L$ be the cokernel of this map and consider the exact sequence

$$
\left(\Pi R_{i}\right) \bigotimes_{I} S \longrightarrow \Pi S_{i} \longrightarrow L \longrightarrow 0,
$$

where $S_{i} \simeq S$ for each $i$. Tensor this sequence with $T$ over $S$ and get the following commutative diagram:

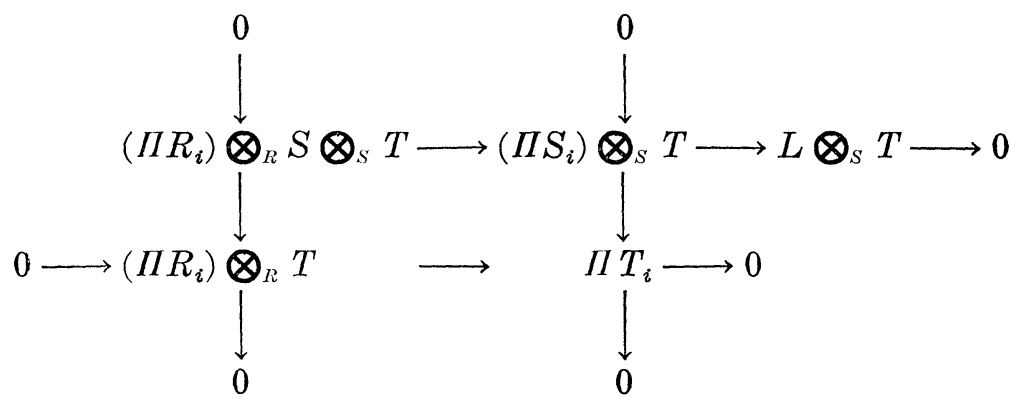

Since the two verticle columns are exact and the bottom row is exact [3, Exercise $9(\mathrm{a})$, p. 43], we have $L \otimes_{S} T=0$. Hence, $L$ is a flat $S$-module with torsion [11, Théorème (1.2.4).] Therefore, $L=0$.

COROLLARY 14. Let $R$ be coherent and let $T$ be a proper overring of $R$ which is finitely generated as an $R$-module. The following are equivalent:

(a) $R \varsubsetneqq T$ is very finite.

(b) Each domain $S, R \subseteq S \subseteq T$, is a finitely presented $R$ module.

(c) Each domain $S, R \subseteq S \subseteq T$, is coherent.

(d) $T$ is finitely presented as a module over each subdomain of $T$ containing $R$.

Proof. (a) $\Rightarrow$ (b) follows as in part of the proof of Proposition $9(\mathrm{~b})$, and $(\mathrm{b}) \Rightarrow(\mathrm{c})$ is immediate from [12, Corollary 1.5]. Assume 
(c) and let $S$ be a subdomain of $T$ containing $R$. Arguing as in Proposition $9(\mathrm{~b})$, we deduce that $T$ is a finitely presented $S$-module, and so $(\mathrm{c}) \Rightarrow(\mathrm{d})$. Finally $(\mathrm{d}) \Rightarrow$ (a) by Lemma 13 .

The next theorem is a partial answer to a question of Vasconcelos, i.e., if $R$ is a local, 1-dimensional coherent domain, is $\bar{R}$ Prüfer? Note that if we also assume $R$ is integrally closed, then $R$ is Prüfer [15, Theorem 1].

THEOREM 15. If $R$ is local, 1-dimensional, coherent, and if $R$ has a very finite extension, then $R$ is Noetherian.

Proof. Assume $R \varsubsetneqq T$ is a very finite extension, and let $M_{1}, \cdots, M_{t}$ be the maximal ideals of $T$ [3, Proposition 3, p. 329]. Let $N$ denote the maximal ideal of $R$. We consider 2 cases:

Case 1. $t>1$. Let $J$ denote the Jacobson radical of $T$, and consider the overring $R+J$ of $R$. Let us observe that $R+J$ is a local domain with maximal ideal $J$ and that $R+J$ is coherent by Corollary 14. Hence, [17, Proposition 1] implies that $J$ is a finitely generated $R+J$-module and thus $R+J$ is Noetherian by Cohen's theorem. (One could also deduce that $J$ is a finitely generated $R+J$-module by realizing that $J$ is a finite intersection of conductors of the form $(a: b)$.) We now apply Eakin's theorem [10, Proposition (6.4.9)] to get $R$ Noetherian.

Note that the proof of case (1) holds when $t \geqq 1$ and $R+J \varsubsetneqq T$.

Case 2. $t=1$ and $T=R+M$. We claim that $N T \neq M$. Assume $N T=M$, and note that $T=R+N T$. But Nakayama's lemma implies that $T=R$, a contradiction. Hence $N T \varsubsetneqq M$ and thus $R+N T \neq T$. Since $R+N T$ is a local domain with maximal ideal $N T$ dominating $N$, the maximal ideal of $R$, we argue as in Case 1 to obtain the desired conclusion.

We globalize Theorem 15 in the following corollary.

Corollary 16. If $R$ is 1-dimensional, coherent, and if for each $P \in \operatorname{Spec}(R)$ where $R_{P}$ is not integrally closed, $R_{P}$ has a very finite extension, then

(a) $R_{P}$ is either a Noetherian domain or a valuation domain for each $P \in \operatorname{Spec}(R)$.

(b) $\bar{R}$ is a Prüfer domain.

Proof. Part (a) is a consequence of Theorem 15 and the remark preceding that theorem. As for (b), let $M$ be a maximal ideal of 
$\bar{R}$ and consider $R_{M \cap R} \subseteq \bar{R}_{M}$. If $R_{M \cap R}$ is a valuation domain, then $\bar{R}_{M}$ is a valuation domain, and if $R_{M \cap R}$ is a Noetherian domain, then [14, Theorem 93] implies that $\bar{R}_{M}$ is again a valuation domain.

REMARK 17. Let $\left(R_{i}, \phi_{i j}\right)$ be a directed system of coherent rings such that if $i \leqq j$ then $R_{j}$ is $R_{i}$ - flat. Then, it is known [3, Exercise $12 \mathrm{e}, \mathrm{p} .44]$ that $R=\lim _{\rightarrow} R_{i}$ is coherent. Hochster, by carefully choosing a directed system $\left(k\left[\left[U_{1}, \cdots, U_{i}\right]\right], \phi_{i j}\right)$ of domains ( $k$ a field) in a manner similar to [22, Ex. 5.28] satisfying the above criteria, has recently pointed out examples of non-Noetherian, nonPrüfer domains that are local, 1-dimensional and coherent, thus showing that the very finite assumption of Theorem 15 is not a necessary condition.

Secondly, we remark that the proof of Theorem 15 combined with the Chinese Remainder Theorem shows that if $R$ is local, coherent and if $R$ has a very finite extension, $R \varsubsetneqq T$, where $T$ is not local, then the maximal ideals of $T$ are finitely generated.

We complete this paper by studying conditions on $R$ and its overrings which force each overring to be of finite type over $R$. Our results in this direction deal with $L H$-domains. See [16, §4] for workable characterizations of $L H$-domains. We first give a useful lemma which generalizes [5, Corollary 4] and [14, Exercise 15, p. 74].

Lemma 18. Let $R$ be coherent and $T$ an overring of $R$. If $R$ is integrally closed in $T$ and if $R \subseteq T$ satisfies going-down, then $R \subseteq T$ is flat.

Proof. Let $M$ be an arbitrary maximal ideal of $T$ and note that $R_{M \cap R}$ is coherent, $R_{M \cap R}$ is integrally closed in $T_{R \backslash M \cap R}$ and $R_{M \cap R} \subseteq$ $T_{R \backslash M \cap R}$ satisfies going-down. Thus $R_{M \cap R} \subseteq T_{R \backslash M \cap R}$ satisfies $L O$ and therefore $R_{M \cap R}=T_{R \backslash M \cap R}$ [15, Theorem 2]. The proof of [20, Theorem 2] shows that $R \subseteq T$ is flat.

LeMMA 19. Let $R$ be open. If $R \subseteq T$ is a flat extension, where $T$ is an overring of $R$, then $T=R[1 / a]$, for some a in $R$. (Hence, $T$ is finitely presented as an $R$-algebra.)

Proof. Since $R$ is open and $R \subseteq T$ is an $i$-extension [16, Remark 2.4], we have that $T$ is open [16, Corollary 3.11], and hence semilocal. Aping the proof of Theorem 2, (a) $\Rightarrow(b)$, we obtain the desired conclusion. 
THEROEM 20. If $R$ is a coherent $L H$-domain and $R$ is integrally closed or $R \varsubsetneqq \bar{R}$ is very finite, then each overring of $R$ is a finitely presented $R$-algebra.

Proof. If $R$ is integrally closed, then $R$ is an open Prüfer domain [16, Lemma 4.13], and so Theorem 2 handles this part. Assume $R \varsubsetneqq \bar{R}$ is very finite, and let $T$ be a proper overring of $R$. Denote the integral closure of $R$ in $T$ by $^{\prime} R^{\prime}$. We may assume $R^{\prime} \varsubsetneqq$ T. Observe that $R^{\prime}$ is coherent [12, Corollary 1.5] and $R^{\prime}$ is open [16, Corollary 3.11]. Lemma 18 shows that $R^{\prime} \varsubsetneqq T$ is flat extension and Lemma 19 combined with the first part of the proof of Proposition $9(\mathrm{~b})$ completes this proof.

REMARK AND EXAMPLeS 21. Example 4 shows that the nonintegrally closed converse of Theorem 20 is not generally true. Example 4 combined with [17, Example 16] and Proposition 9(b) provides us with a nontreed (hence non- $L H$ ) coherent domain, each of whose overrings are finitely presented as $R$-algebras.

We now indicate the significance of the $L H$ assumption in Theorem 20. If we do not assume $R$ is an $L H$-domain yet let the other assumptions remain the same, (coherence is a necessary condition, Converse 10) then the desired finiteness conclusion does not necessarily follow. For example, let $R$ be a 2-dimensional, integrally closed Noetherian domain. Theorem 2 shows that $R$ has a nonfinite type overring. Similarly, let $R$ be a 2-dimensional Noetherian domain such that $R \varsubsetneqq \bar{R}$ is very finite. Proposition 1(c) shows that $R$ must have a nonfinite type extension. (The quotient field of $R$ is an easy example of such an extension.)

Although Theorem 20 does not admit a converse, we can view it as a characterization of those $L H$-domains satisfying the desired finiteness condition. More specifically:

COROLLARY 22. Let $R$ be an LH-domain. Each overring of $R$ is finitely presented as an $R$-algebra if and only if

(a) $R$ is integrally closed; or

(b) $R$ is coherent and $R \varsubsetneqq \bar{R}$ is very finite.

Another variant on the same theme is as follows:

Proposition 23. Let $R$ be 1-dimensional. Each overring of $R$ is finitely presented as an R-algebra if and only if

(a) $R$ is an open Prüfer domain; or 
(b) $R$ is semilocal, coherent, and $R \varsubsetneqq \bar{R}$ is a very finite extension.

Proof. The "only if" part follows from Proposition 1(c) and Proposition 1(a) with Converse 10. Theorem 2 shows that condition (a) is sufficient. Assume (b). Let $T$ be a proper overring of $R$ and let $R^{\prime}$ denote the integral closure of $R$ in $T$. We may assume $R^{\prime} \varsubsetneqq T$. Since $R^{\prime}$ is 1-dimensional, coherent and $R^{\prime}$ is integrally closed in $T$, an appeal to Lemma 18 proves that $R^{\prime} \varsubsetneqq T$ is a flat extension. By [16, Theorem 3.16] we deduce that $R^{\prime}$ is open, and hence an application of Lemma 19 completes the proof.

REMARK 24. Note that (b) in Proposition 23 combined with the 1-dimensional hypothesis and [16, §4] implies that such an $R$ is a coherent $L H$-domain (compare with Remark 21). Hence Proposition 23 can be viewed as a corollary of Corollary 22 . It is also interesting to note that two of the conditions in (b) in conjunction with the 1-dimensional assumption satisfy the hypotheses of Corollary 16. More specifically, if $R$ is 1-dimensional, coherent, and $R \varsubsetneqq \bar{R}$ is very finite, then we obtain conclusion (a) of Corollary 16 . To see this, it is enough to show that when $R_{P} \varsubsetneqq \overline{R_{P}}$, then $R_{P} \varsubsetneqq \overline{R_{P}}$ is a very finite extension. Clearly, $\bar{R}_{P}$ is a finitely generated $R_{P}$-module. Let $S$ be a domain such that $R_{P} \subseteq S \subseteq \overline{R_{P}}$ and observe that ( $S \cap$ $\bar{R})_{R / P}=S_{R / P}$, hence is a finitely generated $R_{P}$-module. Thus $S$ is a finitely generated $R_{P}$-module, which proves the claim.

We conclude this paper with a corollary to Proposition 23.

CoROLlaRy 25. Let $R$ be a Noetherian domain which is not a field. The following are equivalent:

(a) Each overring of $R$ is of finite type over $R$.

(b) Each overring of $R$ is finitely presented as an algebra over $R$.

(c) $R$ is 1-dimensional, semilocal and $R \varsubsetneqq \bar{R}$ is finite or $R$ is 1-dimensional, semilocal, and integrally closed.

Proof. (a) $\Rightarrow$ (b) by Proposition 9 and (c) $\Rightarrow$ (a) by Proposition 23 and Theorem 2 combined with [16, Proposition 3.20]. Assume (b). If $R$ is integrally closed, then an application of Theorem 2 and [16, Proposition 3.20] gives the second condition in part (c), so assume $R \varsubsetneqq \bar{R}$. By assumption $R \varsubsetneqq \bar{R}$ is very finite and that $R$ is semilocal and 1-dimensional follows from Proposition 1(a) and (c).

I am grateful to Wolmer Vasconcelos for his valuable assistance during the preparation of this paper. 


\section{REFERENCES}

0. S. Abhyankar, On the valuations centered in a local domain, Amer. J. Math., 78 (1956), 321-348.

1. E. Bastida and R. Gilmer, Overrings and divisorial ideals of rings of the form $D+M$, Michigan Math. J., 20 (1973), 79-95.

2. N. Bourbaki, Algebra I, Addison-Wesley, Reading, Mass., 1973.

3. — Commutative Algebra, Addison-Wesley, Reading, Mass., 1972.

4. S. U. Chase, Direct products of modules, Trans. Amer. Math. Soc., 97 (1960), 457-519.

5. D. E. Dobbs, On going-down for simple overrings, Proc. Amer. Math. Soc., 39 (1973), 515-519.

6. D. E. Dobbs and I. J. Papick, When is $D+M$ coherent? Proc. Amer. Math. Soc., 56 (1976), 51-54.

7. D. Ferrand et J. P. Olivier, Homomorphismes Minimaux d'Anneaux, J. of Algebra, 16 (1970), 461-471.

8. R. W. Gilmer, Jr. and W. J. Heinzer, Intersections of quotient rings of an integral domain, J. Math. Kyoto Univ., 7 (1967), 133-150.

9. B. Greenberg, Coherence in cartesian squares, submitted for publication.

10. A. Grothendieck and J. A. Dieudonné, Eléments de Géométrie Algébrique, SpringerVerlag, New York, 1971.

11. L. Gruson and M. Raynaud, Critères de platitude et de projectivité, Inventiones Math., 13 (1971), 1-89.

12. M. E. Harris, Some results on coherent rings, Proc. Amer. Math. Soc., 17 (1966), 474-479.

13. B. Iversen, Generic Local Structure in Commutative Algebra, Lecture Notes in Math., Vol. 310, Springer-Verlag, New York, 1973.

14. I. Kaplansky, Commutative Rings, Allyn and Bacon, Boston, Mass., 1970.

15. S. McAdam, Two conductor theorems, J. Algebra, 23 (1972), 239-240.

16. I. J. Papick, Topologically defined classes of going-down domains, Trans. Amer. Math. Soc., 219 (1976), 1-37.

17. - Coherent overrings, Canad. Math. Bull., to appear.

18. G. Picavet Sur les anneaux commutatifs dont tout idéal premier est de Goldman, C. R. Acad. Sc. Paris, t. 280 (30 juin 1975), Serie A, 1719-1721.

19. M. Raynaud, Anneaux Locaux Henséliens, Lecture Notes in Math., Vol. 169, Springer-Verlag, New York, 1970.

20. F. Richman, Generalized quotient rings, Proc. Amer. Math. Soc., 16 (1965), 794-799. 21. W. Vasconcelos, The ג-dimension of a ring, Conference on Commutative Algebra1975, Queen's Papers in Pure and Applied Mathematics, No. 42, (1975).

22. - The Rings of Dimension Two, Lecture Notes in Pure and Applied Math., Vol. 22, Marcel Dekker, New York, 1976.

Received September 2, 1977 and in revised form February 15, 1978.

ADELPHI UNIVERSITY

GARDEN CitY, NY 11530

Current address: University of Missouri Columbia, MO 65211 


\section{PACIFIC JOURNAL OF MATHEMATICS}

\section{EDITORS}

RICHARD ARENS (Managing Editor)

University of California

Los Angeles, California 90024

C. W. CurTis

University of Oregon

Eugene, OR 97403

C. C. MOORE

University of California

Berkeley, CA 94720
J. DUGUNDJI

Department of Mathematics University of Southern California Los Angeles, California 90007

R. FinN AND J. Milgram Stanford University Stanford, California 94305

\section{ASSOCIATE EDITORS}

E. F. BECKENBACH

B. H. NeumanN

F. WOLF

K. YoSHIDA

\section{SUPPORTING INSTITUTIONS}

UNIVERSITY OF BRITISH COLUMBIA CALIFORNIA INSTITUTE OF TECHNOLOGY UNIVERSITY OF CALIFORNIA MONTANA STATE UNIVERSITY UNIVERSITY OF NEVADA, RENO NEW MEXICO STATE UNIVERSITY OREGON STATE UNIVERSITY UNIVERSITY OF OREGON
UNIVERSITY OF SOUTHERN CALIFORNIA

STANFORD UNIVERSITY

UNIVERSITY OF HAWAII

UNIVERSITY OF TOKYO

UNIVERSITY OF UTAH

WASHINGTON STATE UNIVERSITY

UNIVERSITY OF WASHINGTON 


\section{Pacific Journal of Mathematics \\ Vol. 78, No. $1 \quad$ March, 1978}

Simeon M. Berman, A class of isotropic distributions in $\mathbf{R}^{n}$ and their

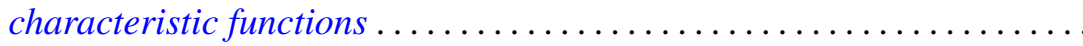

Ezra Brown and Charles John Parry, The 2-class group of biquadratic fields.

II ........................................ 11

Thomas E. Cecil and Patrick J. Ryan, Focal sets of submanifolds ....... 27

Joseph A. Cima and James Warren Roberts, Denting points in $B^{p} \ldots \ldots \ldots 41$

Thomas W. Cusick, Integer multiples of periodic continued fractions . . . . . 47

Robert D. Davis, The factors of the ramification sequence of a class of

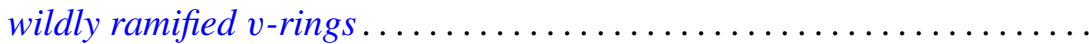

Robert Martin Ephraim, Multiplicative linear functionals of Stein

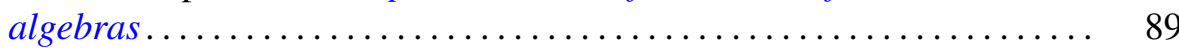

Philip Joel Feinsilver, Operator calculus . .................... 95

David Andrew Gay and William Yslas Vélez, On the degree of the splitting field of an irreducible binomial ..........................

Robert William Gilmer, Jr. and William James Heinzer, On the divisors of

monic polynomials over a commutative ring ..................

Robert E. Hartwig, Schur's theorem and the Drazin inverse .............

Hugh M. Hilden, Embeddings and branched covering spaces for three and four dimensional manifolds ............................

Carlos Moreno, The Petersson inner product and the residue of an Euler product. ...

Christopher Lloyd Morgan, On relations for representations of finite groups....

Ira J. Papick, Finite type extensions and coherence

$\mathrm{R}$. Michael Range, The Carathéodory metric and holomorphic maps on a class of weakly pseudoconvex domains ................

Donald Michael Redmond, Mean value theorems for a class of Dirichlet series

Daniel Reich, Partitioning integers using a finitely generated semigroup ...

Georg Johann Rieger, Remark on a paper of Stux concerning squarefree

numbers in non-linear sequences

Gerhard Rosenberger, Alternierende Produkte in freien Gruppen ..

Ryōtarō Satō, Contraction semigroups in Lebesgue space

Tord Sjödin, Capacities of compact sets in linear subspaces of $\mathbf{R}^{n}$

Robert Jeffrey Zimmer, Uniform subgroups and ergodic actions of exponential Lie groups......................... 